\title{
Can a dietary supplement prevent pre-eclampsia?
}

\author{
L-arginine with vitamins show promise, but there are good grounds for caution
}

\author{
Liam Smeeth professor of clinical epidemiology ${ }^{1}$, David Williams obstetric physician ${ }^{2}$ \\ ${ }^{1}$ Department of Non-communicable Disease Epidemiology, London School of Hygiene and Tropical Medicine, London WC1E 7HT, UK; ${ }^{2}$ Institute \\ for Women's Health, University College London Hospital, London, UK
}

Pre-eclampsia is a pregnancy syndrome that is defined as the gestational onset of hypertension and proteinuria; it affects $2-8 \%$ of first time pregnant mothers and has a marked international variation. ${ }^{1}$ The highest incidence of maternal mortality as a result of pre-eclampsia is in Latin America and the Caribbean, where it accounts for a quarter of maternal deaths. ${ }^{2}$ Most of these maternal deaths are caused by uncontrolled hypertension and multi-organ failure. A simple, cheap, and safe intervention to prevent pre-eclampsia would have a major impact on global health. In the linked randomised trial (doi:10.1136/bmj.d2901), Vadillo-Ortega and colleagues assess whether supplementation during pregnancy with L-arginine (the substrate for synthesis of the vasodilatory gas, nitric oxide) and antioxidant vitamins in a medical food reduces pre-eclampsia in a high risk population.

Despite its simple definition, pre-eclampsia is a complex syndrome with multiple causes that can present with a variety of clinical features. For this reason, a single treatment is unlikely to be effective in all women at risk. As our understanding of pre-eclampsia improves, new therapeutic options emerge. Over the past 20 years, low dose aspirin and calcium supplements have had partial success in preventing the development of pre-eclampsia. Other attempts at preventing pre-eclampsia have been disappointing, in particular the use of antioxidant vitamins. ${ }^{4}$

For a healthy pregnancy outcome blood flow must increase to almost all maternal organs, in particular the uterus and placenta. The maternal vasculature dilates in response to increased activity of the endothelial enzyme nitric oxide synthase. ${ }^{5}$ This enzyme produces the vasodilator substance nitric oxide after stereospecific oxidation of L-arginine. Until recently, it was thought that L-arginine circulated in such abundance that it could not be a rate limiting factor for endothelial production of nitric oxide. However, women with pre-eclampsia have endothelial damage and raised plasma concentrations of a competitive inhibitor of nitric oxide synthase, asymmetrical dimethyl arginine (ADMA). ${ }^{6}$ In vitro, nitric oxide synthase activity is inhibited by intracellular ADMA and rescued by L-arginine supplementation. ${ }^{7}$ Therefore, L-arginine supplementation in pregnancy could possibly overcome nitric oxide synthase inhibition, improve maternal endothelial function, and reduce the risk of pre-eclampsia.

In Vadillo-Ortega and colleagues' trial, women at high risk of pre-eclampsia were randomised to three groups: daily food bars containing both L-arginine and antioxidant vitamins; bars containing only vitamins; and bars with neither L-arginine nor vitamins (placebo). ${ }^{3}$ The intervention started at around 20 weeks' gestation and continued until delivery. The proportion of women developing pre-eclampsia was $30.2 \%$ in the placebo group, $22.5 \%$ in the vitamin only group, and $12.7 \%$ in the L-arginine plus vitamin group. The risk ratio was 0.74 (95\% confidence interval 0.54 to 1.02 ) when comparing the vitamin only group with the placebo group and 0.42 ( 0.28 to 0.62$)$ for the L-arginine plus vitamin group versus the placebo group. L-arginine is a widely available food supplement so the implications of this result could be profound. However, several concerns suggest that a cautious interpretation is warranted.

Firstly, it is not clear how the combination of antioxidant vitamins and L-arginine led to a reduction in pre-eclampsia. Crucially, the effects of L-arginine alone were not studied. A previous trial of L-arginine supplementation for pregnant women with chronic hypertension showed less need for antihypertensive agents, but no reduction in the incidence of superimposed pre-eclampsia. ${ }^{8}$ Furthermore, women who go on to develop pre-eclampsia have been found to have higher, not lower, plasma L-arginine concentrations. ${ }^{9}$ High L-arginine concentrations may counteract raised ADMA values, but other vasoconstrictor effects may persist in women who are vulnerable to pre-eclampsia. ${ }^{9}$ Vadillo-Ortega and colleagues did not measure ADMA concentrations. ${ }^{3}$

Secondly, the primary outcome was classified in a binary manner. The difference in systolic blood pressure between the groups (L-arginine plus vitamins $v$ placebo) was $5 \mathrm{~mm} \mathrm{Hg}$ or less. The large reduction in relative risk was partly driven by a small number of women just crossing the diagnostic blood pressure threshold for pre-eclampsia. Such a simple divide is a poor reflection of the underlying biology: pre-eclampsia is not an "all or nothing" disease, and a more nuanced approach suggests quite modest effects overall. 
A third concern relates to the generalisability of the findings to women at lower risk and to other settings. Most of the women in the trial $(95 \%)$ had a previous pregnancy affected by pre-eclampsia. Even for such a high risk group, the rate of pre-eclampsia in the study population was exceptionally high at $30 \%$, compared with $15 \%$ in a similar group in Sweden. ${ }^{10}$ Beneficial effects of dietary supplements will generally be most pronounced in people with low levels or deficiency of the food supplement in question. Interestingly, calcium supplements reduce the risk of pre-eclampsia in Latin America but not in North America. ${ }^{11}$

Other aspects of the trial were commendable. Although opaque envelopes are not infallible as a means of concealment, allocation was probably adequately concealed. The study was designed to be blinded, with nothing to suggest that knowledge of the randomised allocation affected ascertainment of the outcome. Outcomes were ascertained for all women randomised, and analysis was on an intention to treat basis. Although non-adherence was common, L-arginine was measured and was higher in woman randomised to supplements.

Although the findings are important, crucial questions remain. What is the mechanism of action of L-arginine and vitamins together; what are the effects (including potential adverse effects) of L-arginine given alone; what are the effects in other settings and populations? This is not the conventional "more research is needed" call. Indeed, a crucial first step before more trials are started would be a rigorous systematic review of the numerous inconsistent strands of evidence relating to L-arginine and its possible effects on pre-eclampsia.
Competing interests: All authors have completed the Unified Competing Interest form at www.icmje.org/coi_disclosure.pdf (available on request from the corresponding author) and declare: no support from any organisation for the submitted work; LS has undertaken consultancy for GSK and DW for Roche, neither of which was related to the content of this editorial; LS is supported by a senior clinical fellowship from the Wellcome Trust.

Provenance and peer review: Commissioned; not externally peer reviewed.

1 Duley L. The global impact of pre-eclampsia and eclampsia. Semin Perinatol 2009;33:130-7.

2 Khan KS, Wojdyla D, Say L, Gulmezoglu AM, Van Look PF. WHO analysis of causes of maternal death: a systematic review. Lancet 2006;367:1066-74.

3 Vadillo-Ortega F, Perichart-Perera O, Espino S, Avila-Vergara MA, Ibarra I, Ahued R, et al. Effect of supplementation during pregnancy with L-arginine and antioxidant vitamins in medical food on pre-eclampsia in high risk population: randomised controlled trial. BMJ 2011;342:d2901.

4 Rumbold A, Duley L, Crowther CA, Haslam RR. Antioxidants for preventing pre-eclampsia. Cochrane Database Syst Rev 2008;1:CD004227.

5 Williams DJ, Vallance PJ, Neild GH, Spencer JA, Imms FJ. Nitric oxide-mediated vasodilatation in human pregnancy. Am J Physiol 1997;272:H748-52.

6 Fickling SA, Williams D, Vallance P, Nussey SS, Whitley GSJ. Plasma concentrations of endogenous inhibitor of nitric oxide synthesis in normal pregnancy and pre-eclampsia. Lancet 1993;342:242-3.

7 Cardounel AJ, Cui H, Samouilov A, Johnson W, Kearns P, Tsai AL, et al. Evidence for the pathophysiological role of endogenous methylarginines in regulation of endothelial NO production and vascular function. J Biol Chem 2007;282:879-87.

8 Neri I, Monari F, Sgarbi L, Berardi A, Masellis G, Facchinetti F. L-arginine supplementation in women with chronic hypertension: impact on blood pressure and maternal and neonatal complications. J Matern Fetal Neonatal Med 2010;23:1456-60.

9 Savvidou MD, Hingorani AD, Tsikas D, Frolich JC, Vallance P, Nicolaides KH. Endothelial dysfunction and raised plasma concentrations of asymmetric dimethylarginine in pregnant women who subsequently develop pre-eclampsia. Lancet 2003;361:1511-7.

10 Hernandez-Diaz S, Toh S, Cnattingius S. Risk of pre-eclampsia in first and subsequent pregnancies: prospective cohort study. BMJ 2009;338:b2255.

11 Hofmeyr GJ, Lawrie TA, Attallah AN, Duley L. Calcium supplementation during pregnancy for preventing hypertensive disorders and related problems. Cochrane Database Syst Rev 2010;8:CD001059.

Cite this as: $B M J 2011 ; 342: \mathrm{d} 2777$ 